\title{
Poster: Using Gini Impurity to Mine Attribute-based Access Control Policies with Environment Attributes
}

\author{
Saptarshi Das \\ IIT Kharagpur, India \\ saptarshidas13@iitkgp.ac.in \\ Jaideep Vaidya \\ Rutgers University, USA \\ jsvaidya@business.rutgers.edu
}

\author{
Shamik Sural \\ IIT Kharagpur, India \\ shamik@cse.iitkgp.ernet.in \\ Vijayalakshmi Atluri \\ Rutgers University, USA \\ atluri@rutgers.edu
}

\begin{abstract}
In Attribute-based Access Control (ABAC) systems, utilizing environment attributes along with the subject and object attributes introduces a dynamic nature to the access decisions. The inclusion of environment attributes helps in achieving a more fine-grained access control. In this paper, we present an ABAC policy mining algorithm that considers the environment attributes and their associated values while forming the rules. Furthermore, we use gini impurity to form the rules. This helps to minimize the number of rules in the generated policy. The experimental evaluation shows that our approach is quite effective in practice.
\end{abstract}

\section{KEYWORDS}

ABAC; Policy mining; Environment attributes; Gini impurity

\section{ACM Reference Format:}

Saptarshi Das, Shamik Sural, Jaideep Vaidya, and Vijayalakshmi Atluri. 2018. Poster: Using Gini Impurity to Mine Attribute-based Access Control Policies with Environment Attributes. In SACMAT '18: The 23rd ACM Symposium on Access Control Models 86 Technologies (SACMAT), June 13-15, 2018, Indianapolis, IN, USA. ACM, New York, NY, USA, 4 pages. https://doi.org/10. $1145 / 3205977.3208949$

\section{INTRODUCTION}

In recent years, $\mathrm{ABAC}$ has emerged as the de-facto standard for providing controlled access to organizational resources in scenarios involving inter-organizational sharing of resources. Additionally, ABAC is capable of providing the benefits of all the existing access control models. Although traditional access control models like Role-based Access Control (RBAC) are adequate in providing controlled access to organizational resources in situations involving a known set of users, they have been shown to be incompetent in providing efficient finegrained access control in dynamic environments. This often

Permission to make digital or hard copies of part or all of this work for personal or classroom use is granted without fee provided that copies are not made or distributed for profit or commercial advantage and that copies bear this notice and the full citation on the first page. Copyrights for third-party components of this work must be honored. For all other uses, contact the owner/author(s).

SACMAT '18, June 13-15, 2018, Indianapolis, IN, USA

(C) 2018 Copyright held by the owner/author(s).

ACM ISBN 978-1-4503-5666-4/18/06.

https://doi.org/10.1145/3205977.3208949 necessitates organizations using traditional access control models to migrate to ABAC.

One of the crucial steps for migrating to $\mathrm{ABAC}$ is to construct a set of ABAC authorization rules, collectively called an ABAC policy. Each rule consists of a conjunction of subject, object and environment attributes along with their corresponding values and an operation permitted by the rule. Since manually developing ABAC policies is difficult and costly [4], computational approaches have been deployed to partially automate the process of developing an ABAC policy from a given Access Control Matrix (ACM). Although there exist many ABAC policy mining algorithms, none of these consider the environment attributes and their associated values. Inclusion of environment attributes not only gives a dynamic nature to the access decisions, it also helps in achieving more fine-grained access control.

In this context, we propose a policy mining algorithm that takes an ACM as input and aims to generate an ABAC policy having minimum number of rules. From a broad perspective, as there can be only two possible outcomes of an access request, i.e., a yes or a no, it is possible to classify the accesses given in the ACM on the basis of the attribute data of the subject, object, and environment attributes. We recursively find the attribute data which affects the access decisions most using gini impurity [1] and construct the rules.

While there is some work on mining ABAC policies [3] [5], and also on constrained versions of the ABAC policy mining problem [2], to the best of our knowledge, this is the first attempt towards formulating and solving ABAC policy mining problem which involves environment attributes.

\section{ABAC POLICY SPECIFICATION}

An ABAC system comprises a set of subject attributes $(S A)$, object attributes $(O A)$, environment attributes $(E A)$ and a set of possible operations $(O P)$. Each attribute $a \in$ $S A \cup O A \cup E A$ has a set $\left(A V_{a}\right)$ of possible values. For a set $(S)$ of users, the attribute data for all $s \in S$ is represented using a matrix $(S V)$ of size $|S| \times|S A|$, where each row corresponds to a subject $s \in S$ and each column corresponds to an attribute $a \in S A$. Each $S V[i][j]$ contains the value of the $j^{\text {th }}$ attribute of the $i^{\text {th }}$ subject. For instance, Table 1 shows a subject attribute data matrix from which we can see that Alice is a professor and Bob is a student of the department of CSE. The matrices $O V$ and $E V$ are defined 


\begin{tabular}{l|l|l} 
Subject & Designation & Department \\
\hline Alice & Professor & CSE \\
Bob & Student & CSE \\
\hline
\end{tabular}

Table 1: Subjects and their attributes with associated values

similarly for object attribute data and environment attribute data, respectively. For the sake of brevity, in this work we consider only one operation named as access. However, multiple operations can easily be incorporated in the present work. An ABAC authorization rule is represented as a 4-tuple $\langle S C, O C, E C, O P\rangle$, where $S C, O C, E C$ and $O P$, respectively represent a set of subject attribute data, object attribute data, environment attribute data and the name of an operation. For instance, a rule of the form $\langle\{$ Designation $=$ Student $\},\{$ Type $=$ Assignment $\},\{$ Location $=$ Lab $\}$, view $\rangle$ conveys that any Student can view documents of type Assignment when inside the $L a b$.

\section{ABAC POLICY MINING (APM) PROBLEM}

The policy mining problem takes an ACM as input. In this scenario, the ACM is a 3-dimensional matrix of size $|S| \times$ $|O| \times|E|$ where, each dimension contains the subjects, objects and the environments, respectively. An element $A C M[i][j][k]$, also represented as $\left(s_{i}, o_{j}, e_{k}\right)$ and referred as an access, is 1 , if the $i^{\text {th }}$ subject can access the $j^{\text {th }}$ object in the $k^{\text {th }}$ environment or 0 , otherwise.

\subsection{Problem Definition}

Definition 3.1. ABAC Policy Mining (APM) Problem: Given a set of subjects $S$, a set of objects $O$, a set of environments $E$, a subject attribute matrix $S V$, an object attribute matrix $O V$, an environment attribute matrix $E V$, and an ACM $A$, construct an ABAC policy $P$ such that the following conditions are met: ( $i$ ) each entry in the $\mathrm{ACM} A$ is covered by at least one rule in $P$ (ii) no extraneous access is permitted (iii) number of rules in $P$ is minimum.

\subsection{Solving APM involving Environment Attributes}

In [5], the decision version of APM (D-APM) is proved to be a NP-Complete problem. Thus, it is unlikely that a deterministic polynomial time algorithm exists for solving D-APM. We solve D-APM using a greedy heuristic which involves computing the gini impurity of each attribute data. First, from the given subject, object, and environment attribute data matrices, we construct the attribute data for each possible access. For a given access $(s, o, e)$, the attribute data associated with $s, o$ and $e$ are concatenated along with the corresponding access decision from the access control matrix. This procedure is given in Algorithm 1.

After obtaining the access-data, we construct the ABAC policy. The algorithm for our proposed approach for policy mining is given in Algorithm 2. First, we compute the gini impurity for each of the attribute data to select the attribute data that best divides the accesses into yes and no decisions (Lines 2-6). For instance, Table 2 consists of attribute data

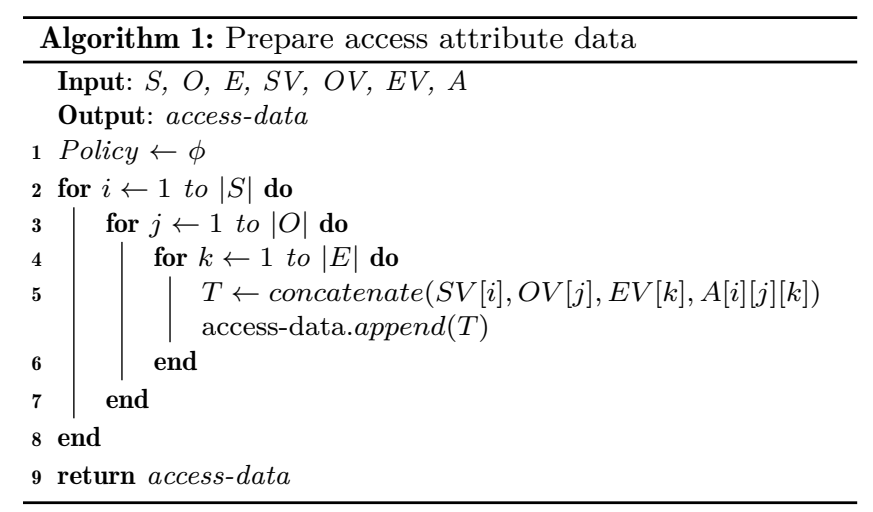

\begin{tabular}{l|l|l} 
Department & Designation & Decision \\
\hline$C S E$ & Professor & no \\
$C S E$ & Professor & no \\
$C S E$ & Professor & no \\
$C S E$ & Professor & no \\
ECE & Professor & no \\
ECE & Professor & yes \\
ECE & Student & yes \\
$C S E$ & Student & yes \\
ECE & Professor & yes \\
$C S E$ & Professor & yes \\
\hline
\end{tabular}

Table 2: Sample access data with decisions

and their corresponding access decisions. There are 2 Departments (Dept.) namely, ECE and CSE and two Designations (Desg.) namely, Professor (Prof.) and Student (Stu.). First, considering the attribute Dept., proportions of ECE and $C S E$ are $\frac{4}{10}$ and $\frac{6}{10}$, respectively. We compute the gini coefficient for $\langle D e p t .=E C E\rangle$. From Table 2, we can see that out of all the instances of ECE, $\frac{3}{4} t h$ and $\frac{1}{4} t h$ instances correspond to yes and no decsions, respectively. Gini coefficient (GC) for an attribute $a$ having value $v$ is calculated as:

$$
G C(a=v)=1-P_{y e s}{ }^{2}-P_{n o}{ }^{2}
$$

Where, $P$ yes is the probability of getting a yes decison when value of attribute $a$ is $v$. $P_{n o}$ is similarly defined. Therefore, gini coefficient for $<$ Dept. $=E C E>$ is:

$$
G C(\text { Dept. }=E C E)=1-\left(\left(\frac{3}{4}\right)^{2}+\left(\frac{1}{4}\right)^{2}\right)=0.375
$$

Similarly, the gini coefficient for $<$ Dept. ! =ECE $>$ which in this example, is same as $<$ Dept. $=C S E>$ is computed as:

$$
G C(\text { Dept. } !=E C E)=1-\left(\left(\frac{2}{6}\right)^{2}+\left(\frac{4}{6}\right)^{2}\right)=0.444
$$

Now, we compute the Gini impurity (GI) for an attribute $a$ having value $v$ as follows:

$G I(a=v)=\left(P_{a=v} * G C(a=v)\right)+\left(P_{a !=v} * G C(a=! v)\right)$

Therefore, GI for $<$ Dept. $=E C E>$ is computed as:

$$
G I(\text { Dept. }=E C E)=\left(\frac{4}{10} * 0.375\right)+\left(\frac{6}{10} * 0.444\right)=0.417
$$




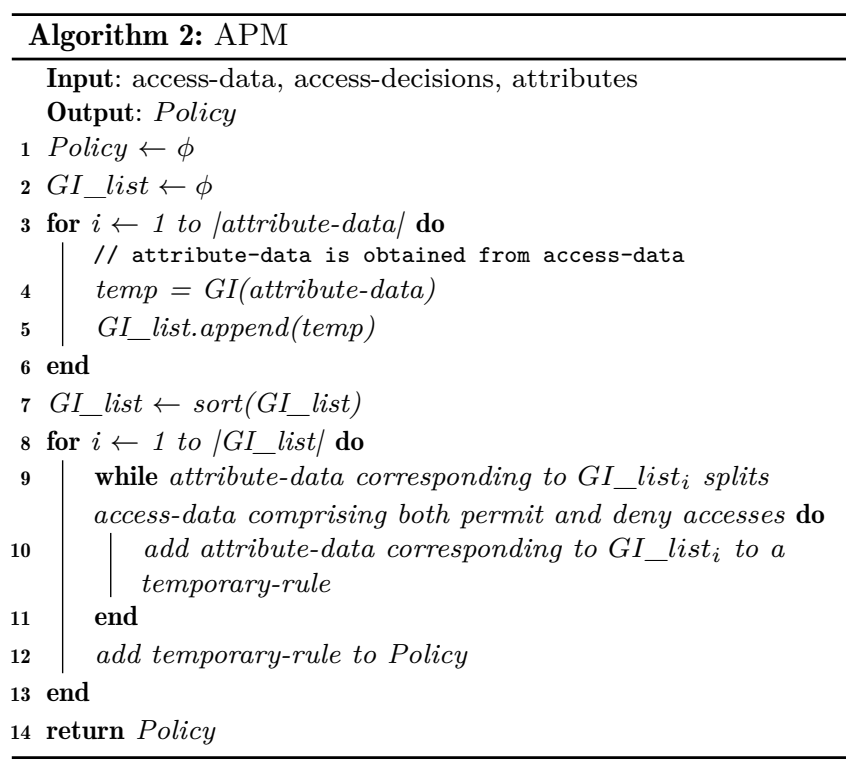

Likewise, $G I($ Desg. $=$ Prof. $)$ is computed to be 0.375. Since, $G I($ Desg. $=$ Prof.$)<G I($ Dept.$=E C E$.$) , we select the for-$ mer as the part of a rule (Line 10). Moreover, we can see that, $G C$ (Desg. ! = Prof.) is computed to be 0, this implies that $<$ Desg. ! = Prof. $>$, i.e., $<$ Desg. $=$ Stu. $>$ contains instances which correspond to a single decision (yes). Thus, $<$ Desg. $=$ Stu.$>$ is a rule. The rest of the accesses are recursively divided following the different gini impurities unless groups of accesses leading to a single decision are obtained (Lines 9-11). Likewise, the rest of the rules are obtained.

\section{EXPERIMENTAL RESULTS}

We implemented our proposed algorithm described in Section 3 in Python 3.6. The implemented algorithm was executed on an Intel i5 processor clocked at $2.70 \mathrm{GHz}$ having 4 GB RAM. We represent the results using a number of subjects $(|S|)$, objects $(|O|)$, number of subject attributes $(|S A|)$, object attributes $(|O A|)$, number of rules formed using the algorithm given in [5] $\left(R_{[5]}\right)$, and our proposed approach $(R)$. First, we evaluated our proposed approach on the data sets given in [5]. As the data sets given in [5] do not consider environment attributes, we execute the algorithm of our proposed approach excluding the environment attributes. From the obtained results, given in Table 3, we can see that the number of rules formed using our proposed approach is less than the number of rules formed in [5]. For smaller data sets like the video library data set, the number of rules formed by our proposed approached is the same. As the data sets grow in size and involve more subject and object attributes along with an increase in the number of subjects and objects, the number of rules formed using our proposed approach is less.

As there are no available data set which contains environment attributes, to evaluate the performance of our proposed approach we synthetically generate various data sets. Table 4 shows the variation in the number of rules in the generated policy and weight of the generated policy with different number of subjects and objects. In our experiments, we have fixed

\begin{tabular}{l|llllll} 
Data set & $|S|$ & $|S A|$ & $|O|$ & $|O A|$ & $R_{[5]}$ & $R$ \\
\hline video library & 12 & 2 & 13 & 2 & 6 & 6 \\
university & 20 & 6 & 34 & 5 & 10 & 9 \\
healthcare & 21 & 6 & 16 & 7 & 11 & 9 \\
project & 16 & 7 & 40 & 6 & 19 & 14 \\
\hline
\end{tabular}

Table 3: Comparison of number of generated rules with [5]

\begin{tabular}{l|llllll}
$\mathrm{S} / \mathrm{O}$ & 100 & 200 & 300 & 400 & 500 & 1000 \\
\hline 20 & 6 & 9 & 12 & 13 & 16 & 19 \\
50 & 10 & 14 & 21 & 24 & 28 & 31 \\
100 & 12 & 15 & 23 & 26 & 31 & 35 \\
200 & 14 & 20 & 28 & 30 & 34 & 41 \\
\hline
\end{tabular}

Table 4: Variation in \#rules with \#subjects and \#objects

the number of subject, object and environment attributes to be 5,5 and 3, respectively. The number of environments was set to 5 . Each cell of Table 4 contains the number of rules in the generated policy for a specific number of subjects and objects. It is observed that the number of rules generated increases with the number of subjects and objects. The proposed approach was also observed to mine an ABAC policy in a considerably less amount of time. For instance, for 200 subjects and 1000 objects, it took only 23.63 seconds to generate a policy comprising 41 rules. Therefore, we have included the environment attributes in policy mining to achieve a more fine-grained access control. Moreover, our proposed approach generates policies having less number of rules as compared to the existing work. A policy having less number of rules is not only compact, but also helps in faster access decisions in case of an actual access request when deployed in an organization.

\section{CONCLUSION}

This paper presents a policy mining algorithm that uses gini impurity to determine the rules for ABAC systems involving environment attributes. Minimizing the number of rules in the generated ABAC policy helps in taking faster access decisions. In the future, we plan to explore other heuristics to further reduce the number of roles as well as attributes used in the generated policy.

\section{ACKNOWLEDGMENTS}

This work was supported by the National Institutes of Health under award R01GM118574 and by the National Science Foundation under award CNS-1624503.

\section{REFERENCES}

[1] L. Breiman. Technical Note: Some Properties of Splitting Criteria. Machine Learning (1997), 41-47.

[2] M. Gautam, S. Jha, S. Sural, J. Vaidya, and V. Atluri. Poster: Constrained Policy Mining in Attribute Based Access Control. Sym. on Acc. Con. Mod. and Tech. (2017), 21-23.

[3] T. Talukdar, G. Batra, J. Vaidya, V. Atluri and S. Sural. Efficient bottom-up Mining of Attribute Based Access Control Policies. IEEE CIC (2017), 339-348.

[4] D. Servos and S. L. Osborn. Current Research and Open Problems in Attribute-Based Access Control. ACM Comp. Sur. (2017), $1-45$

[5] Z. Xu and S. D. Stoller. Mining attribute-based access control policies. IEEE Trans. Dep. and Sec. Com. 12, 5 (2015), 533-545. 